\title{
Implementación de un aula virtual asistida a través de la plataforma Moodle. Caso de la sociedad Mexicana de Criminología Capítulo Nuevo León, A. C.
}

Implementation of an assisted virtual classroom through the Moodle platform.

Case of the Mexican Society of Criminology Chapter New Lion, CSO

Rolando Granados Muñoz ${ }^{1 凶} \underline{\mathrm{ORCID}}$

Fecha correspondencia:

Recibido: 19 de octubre de 2020.

Revisión: 10 de marzo de 2021.

Aceptado: 5 de abril de 2021.

\section{Forma de citar:}

Granados, Rolando. (2021)

"Implementación de un aula virtual

asistida a través de la plataforma

Moodle: Caso de la sociedad

mexicana de criminología capítulo

Nuevo León, A. C." En: Revista CES

Derecho. Vol. 12, No. 1, enero a junio

de 2021, p. 46-57.

Open access

Términos de uso

Licencia creative commons

Etica de publicaciones

Revisión por pares

Gestión por Open Journal System

DOl: http://dx.doi.org/10.21615/

cesder.12.1.3

ISSN: 2145-7719

Sobre los autores:

1. Licenciado en Criminología, con Maestría en Ciencias del

Comportamiento. Profesor de

Criminología y Criminalística.

Director de la revista Archivos de

Criminología, Seguridad Privada

y Criminalística. Especialista

en Formación Docente de

Telebachillerato Comunitario.

\section{Resumen}

En la actualidad el desarrollo de las organizaciones con base en la tecnología es fundamental, en el ámbito educativo y la enseñanza no deja de ser la excepción. El objetivo de este trabajo fue implementar un aula virtual asistida a través de la plataforma Moodle en el área de investigación de la Sociedad Mexicana de Criminología Capítulo Nuevo León, A. C. Se llevó a cabo un diagnóstico FODA, además, se desarrollaron recursos en la plataforma Moodle por medio del sitio de alojamiento mil aulas, obteniendo seis URL, una lección, tres páginas, dos foros, dos exámenes, tres archivos, un taller, una carpeta, una tarea y seis insignias; todos estos recursos programados para ser impartidos en un período de cinco semanas, iniciando del 29 de junio de 2020 al 02 de agosto de 2020. Dos de los participantes nunca ingresaron al aula y al curso; un participante solamente ingresó la primera semana sin realizar actividades. El resto de los participantes fue declinando con el transcurrir del curso, finalizando satisfactoriamente tres participantes sus informes de investigación.

Palabras clave: aprendizaje en línea, aula, educación a distancia, enseñanza programada.

\section{Abstract}

Actually, the development of organizations based on technology is essential, in the educational and teaching fields it is not the exception. The purpose of this article was to implement a virtual classroom assisted through the Moodle platform in the research area of the Mexican Society of Criminology Chapter New Lion, CSO. SWOT diagnosis was carried out, in addition, resources were developed on the Moodle platform through the mil aulas accommodation site, obtaining six URLs, a lesson, three pages, two forums, two exams, three files, a workshop, a folder, one task and six badges; all of these resources scheduled to be taught over a five-week period, beginning June 29, 2020 to August 2, 2020. Two of the participants never entered the classroom and the course; one participant only entered the first week without performing activities. The rest of the 
participants declined over the course of the course, with three participants successfully completing their research reports.

Keywords: classroom, distance education, electronic learning, programmed instruction.

\section{Introducción}

Las tecnologías de la información han formulado nuevos retos no solo para las personas sino para las instituciones, su uso constante implica adquirir nuevas capacidades para forjar un carácter competitivo, eso se traduce en la vigencia y avances hasta alcanzar el objetivo que pretenden. En síntesis, la tecnología y la educación han conseguido hacer un binomio importante para el desarrollo en las sociedades modernas; descritas como sociedades de la información y del conocimiento (Alfonso, 2016; Crovi, 2005; Pérez, Mercado, Martínez \& Mena, 2018; Terrazas \& Silva, 2013), estas sociedades han permitido generar las condiciones óptimas para la incorporación y participación de los individuos en la mejora constante de procesos. Al respecto Gido y Clements (1999) desde hace décadas mencionaban lo siguiente:

"Las nuevas tecnologías se han convertido en un factor importante para casi cada empresa, y seguirán siéndolo. Las computadoras y la tecnología de las comunicaciones han transformado en forma radical el lugar de trabajo y muchos gerentes contemplan este nuevo ambiente como más retador que el anterior" (p. 268).

En ese acontecer el tema de las aulas virtuales no deja de ser una propuesta llamativa que hace posible elevar el potencial de la oferta educativa, en el caso de la plataforma Moodle con sus diversos recursos como chats, foros, cuestionarios, lecciones, talleres, libros, etc., permiten optimizar la gestión de la enseñanza y el aprendizaje con la posibilidad de posicionarse ante un público mayor y más diverso, destacándose por ser ampliamente flexible y cómoda para aquellas personas que no siempre pueden asistir presencialmente a las clases (Bustos \& Coll, 2010; Casano, 2006; Homero, 2017; Llorente, 2007; Marín, Ramírez \& Sampedro, 2011). Este impacto de las tecnologías también es perceptible en las distintas disciplinas o áreas del conocimiento, no deja de ser la excepción en el ámbito de la investigación, haciendo posible que áreas de oportunidad y deficiencias sean aprovechadas convirtiéndolas en fortalezas; se asume que un aula virtual es conveniente pues da acceso a que más usuarios, profesionales, nuevas líneas de investigación, otras instituciones, etc., mantengan una relación con dicha organización y se fortalezcan sus métodos de enseñanza. Como lo detallan Lagunes et al. (2015) en su estudio sobre plataformas educativas virtuales, las herramientas que estas plataformas aportan permiten el impulso de la investigación a través de materiales dinámicos y didácticos para fomentar el aprendizaje, mejorando las asesorías y tutorías que son impartidas.

Se ha constatado el impacto que tienen las herramientas de la plataforma Moodle en los estudiantes y en los profesores, específicamente en el liderazgo y en el aprendizaje, incluso en la actitud hacia la tecnología y las formas metodológicas de impartir las clases (Herrera, 2009; Ramírez \& Baraja, 2017; Lechuga, Fernández-Arteaga, Ríos \& Fernández-Serrano, 2014; Martínez-Bahena, Lopez-Escogido, Escamilla-Regis, \& Älvarez-Monroy, 2017; Pérez \& Saker, 2013; Sierra, 2011), aunque, como lo refieren Vargas-Cubero y Villalobos-Torres (2018), para obtener un resultado favorecedor, se 
requiere organización, control y orden en la manera de gestionar las asignaturas, el apoyo y el personal docente. Ha sucedido en varios países y en otras áreas de la ciencia que las aulas virtuales pueden favorecer al desarrollo de redes de información y de procesos, lo que permite un acercamiento entre instituciones y distintos tipos de personas (Rojas, Pérez, Torres \& Peláez, 2014). Siendo al final de cuentas una de las prioridades de las organizaciones, es decir vincularse con otras instituciones. Además de ello, enseñar a utilizar con mesura y de manera adecuada la tecnología.

En contexto de estos hallazgos que se han comentado acerca de la tecnología y la educación, así como las ventajas de las aulas virtuales, se llevó a cabo la planeación de un proyecto para que permitiera mejorar las habilidades digitales de los usuarios e influyera en la actitud de éstos hacia la investigación. Así mismo, para aumentar la producción de conocimiento científico. El problema de investigación planteado se suscita en la Sociedad Mexicana de Criminología Capítulo Nuevo León, A. C., en relación a un problema de investigación, se puede considerar a éste como determinante al investigar, darle forma implica describirlo y cuestionarse acerca de las características que presenta el fenómeno de interés (Trinchet \& Trinchet, 2007), a continuación, antes de hacer énfasis en ello, se hace una breve remembranza de las principales características de la organización.

La Sociedad Mexicana de Criminología Capítulo Nuevo León, A. C. (SOMECRIMNL, 2020) es una organización no lucrativa que desde el año 2008 se ha dedicado a generar avances en áreas forenses, desde sus inicios nace como una entidad enfocada a la investigación y formación; no tiene sedes, pero cuenta con una oficina física, legal y fiscal, se constituye de miembros que no están contratados de tiempo completo, sino que contribuyen en sus tiempos a través de medios digitales, cuando es posible se les otorga apoyo por aportaciones materiales o profesionales y se contrata personal externo para la implementación de proyectos. Para el año 2020 la organización contabiliza 23 miembros, entre ellos tres instituciones y los otros 20 siendo miembros asociados, consejeros, afiliados y especiales. Su giro está circunscrito en lo asistencialista, desarrollo social, cultura y educación.

Sus rubros de acción están enfocados a lo profesional desarrollando distintos estudios y proyectos en temas de violencia, delincuencia y/o criminalidad; además, generando conocimiento y haciendo difusión de este, y colaborando con distintos tipos de instituciones. En lo educativo su objetivo es impartir enseñanza de cursos, talleres, diplomados, conferencias y otras modalidades de enseñanza, el público al que llega es la sociedad y especialmente a la comunidad estudiantil. El innovar en cuestiones tecnológicas es fundamental. El área de investigación se dedica a generar y difundir conocimiento, se considera óptima para implementar un aula virtual. Esta área de investigación cuenta con su propia misión y visión (SOMECRIMNL, 2020).

- Misión. La promoción del conocimiento en las áreas criminológicas, forenses y penales mediante procesos de publicación éticos, claros y sólidos.

- Visión. Ser un órgano de difusión abierto con calidad en el proceso de publicación, mejorando de manera constante, haciendo apertura a los avances y ética formal en las ciencias criminológicas, forenses y penales. 
La problemática de la organización se basa en la gestión o administración del conocimiento, la principal función del área de investigación es la producción de conocimiento científico, el proyecto en sí busca aumentar esa producción acorde a los informes de investigación que sean obtenidos de un taller impartido a través de la plataforma Moodle.

Para realizar dicha gestión del conocimiento es que se adoptó como objetivo general de la investigación:

- Implementar un aula virtual asistida a través de la plataforma Moodle en el área de investigación de la Sociedad Mexicana de Criminología Capítulo Nuevo León, A. C. en un período de cinco semanas.

Los objetivos específicos para alcanzar el principal fueron:

1. Desarrollar contenido y actividades de un taller de elaboración de informes de investigación en las primeras tres semanas antes de ofertar el curso.

2. Programar en la plataforma Moodle el contenido y las actividades desarrolladas usando el sitio de alojamiento gratuito mil aulas.

3. Poner en marcha el taller de elaboración de informes de investigación en un período de cinco semanas.

\section{Método y materiales}

Este trabajo se plantea considerando la técnica casuística de investigación, lo que implica interpretar las cualidades particulares de una realidad única y una forma delimitada tanto de las características propias como puede ser el tiempo y el espacio, así como del diseño, los propósitos, conclusiones o el informe que se obtienen, puede ser de diferentes tipos, incluso puede ser un solo caso o múltiples casos con un método que emplea diversas fuentes y técnicas de recogida de información (Ceballos-Herrera, 2009; Jiménez \& Comet, 2016).

En primera instancia se empleó la metodología o matriz FODA (fortalezas, oportunidades, debilidades, amenazas) para hacer un diagnóstico de la organización, esta es una técnica que se emplea para el análisis o evaluación de problemas en el contexto interno organizacional que ayuda a determinar los factores que impactan a la organización y favorece a la planeación de estrategias que permiten forjar la competitividad (Ponce, 2007; Ramírez, 2009; Villagómez, Mora, Barradas \& Vázquez, 2014). Los datos aportados por la matriz FODA permitieron tener una referencia más precisa acerca de la funcionalidad de la SOMECRIMNL y de esta manera poder seleccionar la población objetivo del taller y la emisión del material por el que éste se constituiría.

Una vez recabados estos datos, las actividades que se llevaron a cabo para su desarrollo fueron las siguientes:

1. Búsqueda documental

2. Generación del contenido y las actividades del taller de elaboración de informes de investigación (lecturas, lecciones, cuestionarios, entregables, foros, vídeos, etc.). 
3. Una vez bien definido el curso, se llevó a cabo su promoción a través de distintos medios (Facebook, Whats App, Messenger).

4. También se hizo la elección del profesor a cargo y los estudiantes.

5. Puesta en marcha del curso (taller).

6. Análisis de los resultados.

7. Elaboración del reporte de resultados para la organización.

Teniendo de referencia la temporalidad de las actividades antes descritas las cuales fueron pautadas por fechas, así como dar seguimiento a los objetivos específicos para dar cumplimiento al objetivo general, lo primero fue hacer el registro en el sitio de alojamiento mil aulas para poder tener acceso a la plataforma Moodle y trabajar en desarrollo de las actividades y recursos que estructuraron el taller. Se hizo una planeación de la estructura del curso y las probables actividades, al mismo tiempo se estuvieron diseñando las imágenes que aparecerían como portada del curso (taller), en las unidades y las insignias que se obtendrían a lo largo del curso. Se realizaron lecturas descargables y los ítems que conformaron las preguntas de cada examen, además de grabar y editar vídeos.

\section{Resultados}

Según los resultados del diagnóstico realizado en la matriz FODA, entre los factores de éxito se refiere que existe una preocupación por la mejora constante en la gestión del conocimiento, ya que incluso dentro de las amenazas se especifica la ignorancia por parte de las personas acerca de temas forenses. Retomado también el contexto de la investigación en donde la misión y visión están centrados en la promoción de conocimiento y mejora en los procesos, pese a que toda esa administración del conocimiento se genera en línea, la situación que enfrenta la organización es establecer una nueva forma al menos para la Sociedad Mexicana de Criminología Capítulo Nuevo León, A. C., el gestionar la producción del conocimiento.

El primer objetivo específico del proyecto consistió en desarrollar contenido y actividades de un taller de elaboración de informes de investigación en las primeras tres semanas antes de ofertar el curso, como ya se hizo mención antes, estas mismas se desglosan en la Tabla 1, en total fueron generados 10 tipos de recursos o actividades. 
Tabla 1. Recursos o actividades de Moodle desarrollados.

\begin{tabular}{|c|c|c|}
\hline Tipo de recurso & Cantidad & Nombre \\
\hline \multirow{6}{*}{ URL } & \multirow{6}{*}{ Seis } & 1. Evaluación del desempeño I (Google forms). \\
\hline & & 2. Evaluación del desempeño II (Google forms). \\
\hline & & 3. ¿Cómo navegar en la plataforma virtual? (Vídeo). \\
\hline & & 4. Presentación del curso (Vídeo). \\
\hline & & 5. Presentación del informe (Vídeo). \\
\hline & & 6. Introducción presentación del informe. \\
\hline Lección & Una & 1. Partes del informe. \\
\hline \multirow{3}{*}{ Página } & \multirow{3}{*}{ Tres } & 1. Contenido del curso \\
\hline & & 2. Introducción a la investigación. \\
\hline & & 3. La importancia del método. \\
\hline \multirow{2}{*}{ Foro } & \multirow{2}{*}{ Dos } & 1. Bienvenida y cronograma de trabajo \\
\hline & & 2. ¿Qué es un informe de investigación? \\
\hline \multirow{2}{*}{ Examen } & \multirow{2}{*}{ Dos } & 1. Examen unidad 1. \\
\hline & & 2. Examen unidad 2. \\
\hline \multirow{3}{*}{ Archivo } & \multirow{3}{*}{ Tres } & 1. La investigación en el ámbito criminológico y criminal. \\
\hline & & 2. La metodología y el informe de investigación. \\
\hline & & 3. Tipos de informes de investigación. \\
\hline Taller & Uno & 1. Mi informe de investigación. \\
\hline Carpeta (folder) & Una & 1. Muestra de informes. \\
\hline Tarea & Una & 1. Informe de investigación final. \\
\hline \multirow{6}{*}{ Insignias } & \multirow{6}{*}{ Seis } & 1. Estudiante súper evaluado. \\
\hline & & 2. Estudiante sabio del cuestionario. \\
\hline & & 3. Estudiante graduado. \\
\hline & & 4. Estudiante entrenado en la lección. \\
\hline & & 5. Estudiante de participación profesional. \\
\hline & & 6. Estudiante aficionado por la lectura. \\
\hline
\end{tabular}

Una vez teniendo la planeación y desarrollo de las actividades se procedió al segundo objetivo específico el cual consistía en programar en la plataforma Moodle el contenido y las actividades desarrolladas usando el sitio de alojamiento gratuito mil aulas. Se hizo la distribución de los recursos o actividades en unidades y se establecieron los tiempos de duración y las fechas del curso. Esta misma programación se muestra en la Tabla 2 con sus respectivas características. 
Tabla 2. Programación de los recursos a través de la plataforma Moodle mil aulas

\begin{tabular}{|c|c|c|}
\hline Organización & Contenido & Temporalidad \\
\hline $\begin{array}{l}\text { Página principal } \\
\text { del curso }\end{array}$ & $\begin{array}{l}\text { a. Bienvenida y cronograma de trabajo } \\
\text { b. Contenido del curso }\end{array}$ & $\begin{array}{l}\text { Del } 29 \text { de junio al } 02 \\
\text { de agosto de } 2020 .\end{array}$ \\
\hline $\begin{array}{l}\text { Evaluación del } \\
\text { desempeñol }\end{array}$ & a. Cuestionario de habilidad digital e investigación & $\begin{array}{l}\text { A partir del } 29 \text { de } \\
\text { junio de } 2020\end{array}$ \\
\hline $\begin{array}{l}\text { Unidad 1. El } \\
\text { informe de } \\
\text { investigación }\end{array}$ & $\begin{array}{l}\text { a. 1.1. Introducción a la investigación } \\
\text { b. 1.2. La investigación en el ámbito criminológico } \\
\text { y criminal } \\
\text { c. 1.3. La importancia del método } \\
\text { d. 1.4. La metodología y el informe de investigación } \\
\text { e. 1.5. ¿Qué es un informe de investigación? } \\
\text { f. Examen Unidad } 1 \text { (cinco minutos) }\end{array}$ & $\begin{array}{l}\text { A partir del } 29 \\
\text { de junio de } 2020 \text {, } \\
\text { pero debiendo de } \\
\text { contestar antes } \\
\text { la Evaluación del } \\
\text { desempeño I }\end{array}$ \\
\hline $\begin{array}{l}\text { Unidad } 2 . \\
\text { Estructura del } \\
\text { informe de } \\
\text { investigación }\end{array}$ & $\begin{array}{l}\text { a. 2.1. Tipos de informes de investigación } \\
\text { b. 2.2. Partes del informe } \\
\text { c. 2.3. Mi informe de investigación } \\
\text { d. Examen Unidad } 2 \text { (seis minutos) }\end{array}$ & $\begin{array}{l}\text { A partir del } 06 \text { de } \\
\text { julio de } 2020\end{array}$ \\
\hline $\begin{array}{l}\text { Unidad } 3 . \\
\text { Presentación } \\
\text { del informe }\end{array}$ & $\begin{array}{l}\text { a. 3.1. Introducción presentación del informe } \\
\text { b. 3.2. Muestra de informes } \\
\text { c. 3.3. Informe de investigación final }\end{array}$ & $\begin{array}{l}\text { A partir del } 27 \text { de } \\
\text { julio de } 2020\end{array}$ \\
\hline $\begin{array}{l}\text { Evaluación del } \\
\text { desempeñol }\end{array}$ & a. Cuestionario de habilidad digital e investigación & $\begin{array}{l}\text { A partir del } 27 \text { de } \\
\text { julio de } 2020\end{array}$ \\
\hline
\end{tabular}

Teniendo certeza de las fechas y de la duración del curso el objetivo específico que prosiguió fue poner en marcha el taller de elaboración de informes de investigación en un período de cinco semanas, para ello, como se mostró en el desarrollo de actividades se hizo la promoción del curso a través de redes sociales especialmente a partir de la página de Facebook de la Sociedad Mexicana de Criminología Capítulo Nuevo León, A. C.

Fueron inscritos un total de 10 participantes, el medio de contacto fue de $40 \%$ por Messenger, el 40\% por Whats App y el 20\% por medio de correo electrónico.

El día 28 de junio de 2020 fueron enviados a través de correo electrónico los usuarios y las contraseñas, además de ello se les hizo llegar el enlace para ingresar al aula. Dos de los participantes nunca ingresaron al aula y al curso; un participante solamente ingresó la primera semana sin realizar actividades. Parte del resto de los participantes fue declinando con el transcurrir del curso, este avance se presenta en la Figura 1 ya que hace referencia a la cantidad de participantes que fueron realizando las actividades.

Según el cuestionario incluido en la Evaluación del desempeño I, es decir cuando todos ingresaron, los participantes cumplen con las siguientes características: 


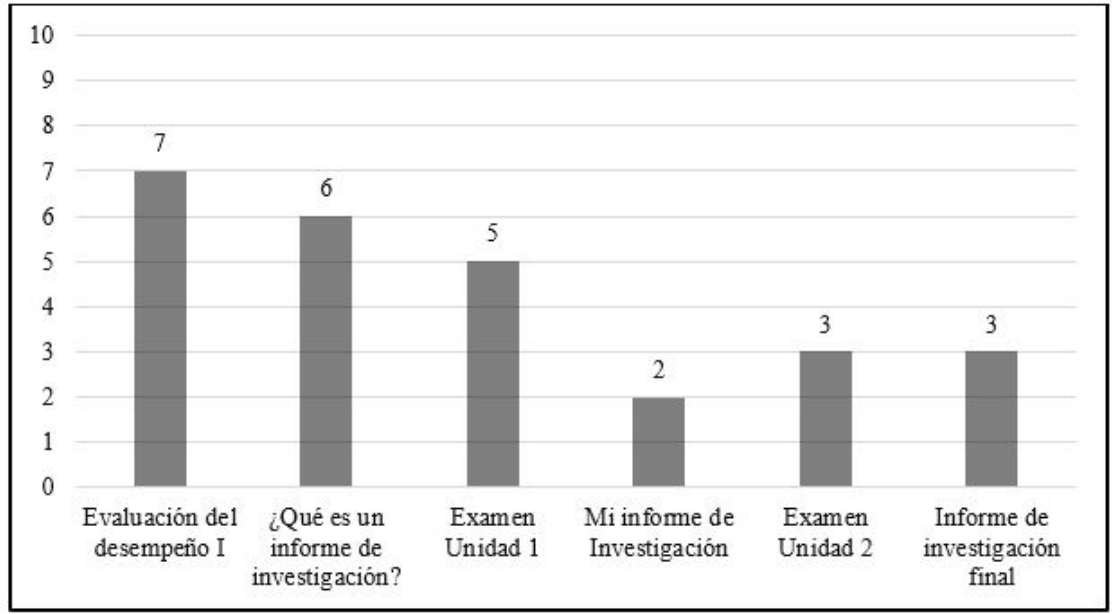

Figura 1. Cantidad de participantes que fueron realizando las actividades.

Provenientes de cinco universidades distintas, del Instituto Universitario del Centro de México (EDUCEM), la Universidad de Guanajuato, La Escuela Potosina Libre de Derecho y una que no fue especificada. Cuatro de Irapuato, uno de San Luis Potosí, uno de Salamanca y uno de Silao. Con un rango de los 19 a los 32 años. La distribución por sexo se presenta en la Figura 2.

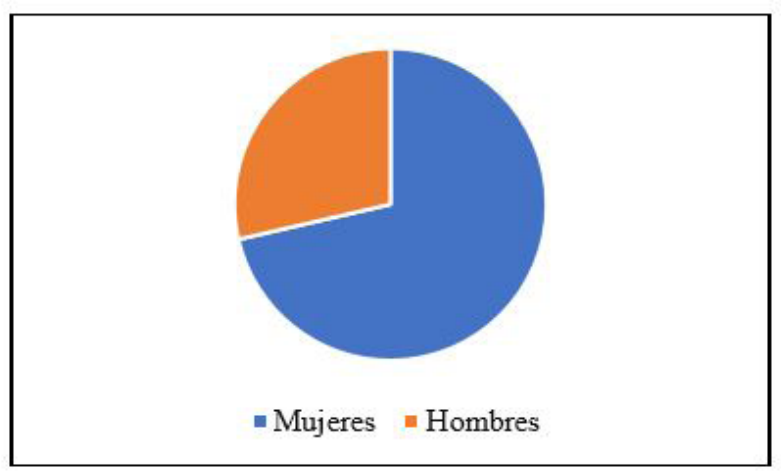

Figura 2. Distribución de participantes por sexo.

El nivel de estudios se presenta en la Figura 3, considerando a los estudiantes de niver superior en el apartado pregrado, a los que contaban con título profesional al apartado grado y los que tenían maestría en el apartado posgrado. 


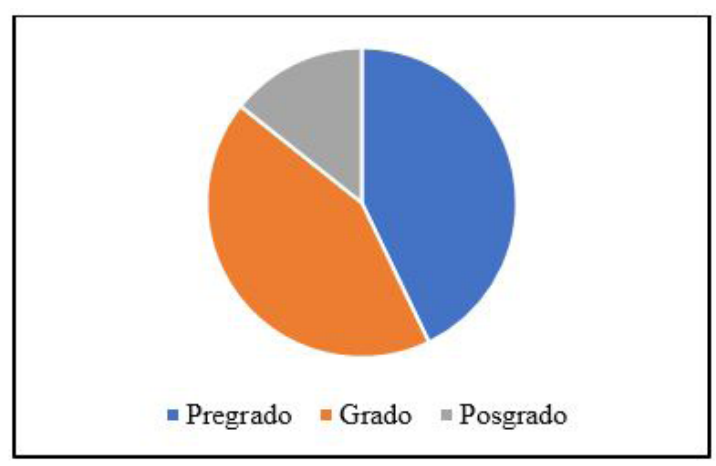

Figura 3. Distribución de los participantes por nivel de estudios.

El avance del curso de cada participante se especifica en la Tabla 3, la cual dispone de las actividades llevadas a cabo que fueron calificadas y las respectivas calificaciones de los participantes. Aquellos estudiantes con retraso de actividades se les suspendió del curso.

Tabla 3. Actividades que cuentan con calificación.

\begin{tabular}{cccccc}
\hline \multirow{2}{*}{ Estudiantes } & \multicolumn{5}{c}{ Actividades } \\
\cline { 2 - 6 } & $\begin{array}{c}\text { ¿Qué es un informe } \\
\text { de investigación? }\end{array}$ & $\begin{array}{c}\text { Examen } \\
\text { Unidad 1 }\end{array}$ & $\begin{array}{c}\text { Mi informe de } \\
\text { investigación }\end{array}$ & $\begin{array}{c}\text { Examen } \\
\text { Unidad 2 }\end{array}$ & $\begin{array}{c}\text { Informe de } \\
\text { investigación final }\end{array}$ \\
\hline Alejandro & 10 & 9 & 40 & 9.3 & $15.80(84.1)$ \\
\hline Carolina & 10 & 4 & 38 & 6.51 & $16.60(75.11)$ \\
\hline Jazmín & 8 & 5.5 & No entregó & 4.07 & $10.20(27.77)$ \\
\hline Lizbeth & 10 & 8.5 & Suspendida & Suspendida & Suspendida \\
\hline María & Suspendida & Suspendida & Suspendida & Suspendida & Suspendida \\
\hline Patricia & Suspendida & Suspendida & Suspendida & Suspendida & Suspendida \\
\hline Paulina & Suspendida & Suspendida & Suspendida & Suspendida & Suspendida \\
\hline Roberto & Suspendida & Suspendida & Suspendida & Suspendida & Suspendida \\
\hline Ruth & 10 & 6.5 & Suspendida & Suspendida & Suspendida \\
\hline Susana & Suspendida & Suspendida & Suspendida & Suspendida & Suspendida \\
\hline
\end{tabular}

\section{Discusión}

En materia criminológica se percibe una renuente y carente importancia por la investigación de parte de las personas, no les interesa mostrar avances de su ciencia ni realizar una trayectoria académica.

Según se muestra en las gráficas y considerando los aspectos desde el desarrollo hasta la puesta en marcha del curso (taller), se debe aclarar que la idea base del taller buscaba preparar a las personas para obtener un producto final, es decir un informe de investigación con posibilidad de publicarse para la revista de la sociedad, realmente no se buscaba cantidad sino calidad. Se buscaba algo similar a la propuesta de Lagunes, Castro, Flores, Ortiz y Lagunes (2015) al hacer presente la importancia de las asesorías en lo referente a la investigación, ya que puede potenciar y favorecer al desarrollo de esta misma. 
El aula virtual es una herramienta sumamente fácil, aunque se tiene a consciencia que para un mejor manejo de los recursos lo ideal hubiera sido una preparación pre ingreso en la que se explicara la manera de funcionar de la plataforma de Moodle antes de que se pusiera en práctica el curso taller, pues eso pudo haber influido en las bajas de los participantes. Es importante considerar lo que ya se ha escrito en el tema de aulas virtuales y sobre todo en apego al área criminológica (Vargas-Cubero \& Villalobos-Torres, 2018), el seguimiento que se les dé a los alumnos, con orden y control mantendrá una parte del éxito de la enseñanza.

Aunque en los comunicados se les hacía hincapié a los participantes en expresar sus dudas realmente ellos se mostraban poco participativos, además de ello en las calificaciones de los exámenes incluso llegaron a comunicarse con la finalidad de hacer saber que les había ido mal.

El objetivo de investigación fue cumplido a totalidad, algo de los más destacado y que puede consultarse en los resultados es la cantidad de recursos o actividades emergentes, además de ello es posible detectar que la plataforma es un medio adecuado para la distribución y aplicación de estas actividades, es decir, se pudo corroborar la funcionalidad de Moodle como una plataforma ideal para desarrollar contenido educativo en línea, y con ello dar crédito a la empleabilidad de las aulas virtuales como ya lo han mencionado algunos autores (Casano, 2006; Homero, 2017; Llorente, 2007; Marín, Ramírez \& Sampedro, 2011) para la flexibilidad y el ajuste a las necesidades de las personas que están con pocas posibilidades de llevar a cabo sus estudios de manera presencial y sobre todo su implementación en las áreas criminológicas.

Aunque no fue posible hacer mediciones del efecto del aula virtual como se había plateado debido a la cantidad de estudiantes, como se tiene registrado en otras investigaciones que persiguen objetivos símiles (Herrera, 2009; Ramírez \& Baraja, 2017; Lechuga, Fernández-Arteaga, Ríos \& Fernández-Serrano, 2014; Martínez-Bahena, Lopez-Escogido, Escamilla-Regis, \& Älvarez-Monroy, 2017; Pérez \& Saker, 2013, Sierra, 2011), las descripciones apuntan a que es un taller que se puede mejorar y con ello hacer una mejor configuración de la plataforma para que la Sociedad Mexicana de Criminología Capítulo Nuevo León, A. C., tenga a disposición una nueva herramienta para gestionar ese conocimiento que en criminología es una necesidad, tan solo en este período de tiempo fue posible obtener al menos dos artículos con posibilidad de publicarse en la revista Archivos de Criminología, Seguridad Privada y Criminalística o en cualquier otra porque cumplen con las características y la calidad de publicación.

Implementar un aula virtual en esta organización permitió ver más allá de la edición y revisión de artículos que es parte del proceso que se lleva a cabo por la revista que la misma sociedad maneja, en vistas a futuro este hallazgo permite establecer que para la misión y visión acerca de difundir conocimiento de calidad, el ofrecer un curso (taller) en esta modalidad hace posible adaptar los artículos de los autores a sus propios estándares.

\section{Referencias}

Alfonso, I. R. (2016). La sociedad de la información, sociedad del conocimiento y sociedad del aprendizaje. Referentes en torno a su formación. Bibliotecas. Anales de Investigación, 12(2), 235-243. 
Bustos, A., \& Coll, C. (2010). Los entornos virtuales como espacios de enseñanza y aprendizaje. Una perspectiva psicoeducativa para su caracterización y análisis. Revista Mexicana de Investigación Educativa, 15(44), 163-184.

Casano, F. (2006). La plataforma de aprendizaje Moodle como instrumento para el trabajo social en el contexto del espacio europeo de la educación superior. Acciones e Investigaciones Sociales, (Ex. 1), 367.

Ceballos-Herrera, F. A. (2009). El informe de investigación con estudio de casos. Magis. Revista Internacional de Investigación en Educación, 1(2), 413-423.

Crovi, D. M. (2005). La sociedad de la información: una mirada desde la comunicación. Ciencia, 56(4), 23-37.

Gido, J., \& Clements, J. P. (1999). Administración exitosa de proyectos. International Thomson Editores.

Herrera, A. F. (2009). Impacto de las aulas virtuales como mediación pedagógica en las asignaturas presenciales de pregrado del departamento de humanidades de la Universidad Militar Nueva Granada. Revista Educación y Desarrollo Social, 3(2), 118-132.

Homero, P. (2017). Plataformas virtuales y su impacto en la educación superior. Explorador Digital, 1(4), https://doi.org/10.33262/exploradordigital.v1i2.318

Jiménez, V. E., \& Comet, C. (2016). Los estudios de casos como enfoque metodológico. Academo Revista de Investigación en Ciencias Sociales y Humanidades, 3(2).

Lagunes, A., Castro, C. A., Flores, M. A., Ortiz, A. F., \& Lagunes, P. (2015). Plataformas educativas virtuales como apoyo a la tutoría de investigación para estudiantes universitarios. Trabajo presentado en la conferencia del XXI Congreso Internacional sobre Educación Bimodal, Colombia.

Llorente, M. C. (2007). Moodle como entorno virtual de formación al alcance de todos. Comunicar, 15(28), 197-202.

Lechuga, M., Fernández-Arteaga, A., Ríos, F., \& Fernández-Serrano, M. (2014). Utilización de entornos virtuales educativos y recursos educativos abiertos (OpenCourseWare) en cursos de ingeniería química de la Universidad de Granada, España. Formación Universitaria, 7(4), 3-14. https://dx.doi.org/10.4067/S0718-50062014000400002

Marín, V., Ramírez, A., \& Sampedro, B. (2011). Moodle y estudiantes universitarios. Dos nuevas realidades del EEES. Profesorado. Revista de Currículum y Formación de Profesorado, 15(1),109-120.

Martínez-Bahena, E., Lopez-Escogido, D., Escamilla-Regis, D., \& Älvarez-Monroy, L. M. (2017). La importancia de las plataformas educativas virtuales como herramienta de apoyo a la educación tradicional. Revista de Tecnología y Educación, 1(1), 16-24. 
Pérez, M. L., \& Saker, A. (2013). Importancia del uso de las plataformas virtuales en la formación superior para favorecer el cambio de actitud hacia las TIC; estudio de caso: Universidad del Magdalena, Colombia. Revista Iberoamericana de Evaluación Educativa, 6(1), 153-166.

Pérez, R., Mercado, P., Martínez, M., Mena, E., \& Partida, J. Á. (2018). La sociedad del conocimiento y la sociedad de la información como la piedra angular en la innovación tecnológica educativa. Revista Iberoamericana para la Investigación y el Desarrollo Educativo, 8(16), 847-870. http://dx.doi.org/10.23913/ride. v8i16.371

Ponce, H. (2007). La matriz foda: alternativa de diagnóstico y determinación de estrategias de intervención en diversas organizaciones. Enseñanza e Investigación en Psicología, 12(1), 113-130.

Ramírez, J. L. (2009). Procedimiento para la elaboración de un análisis FODA como una herramienta de planeación estratégica en las empresas. Ciencia Administrativa, (2), 54-61.

Ramírez, W., \& Baraja, J. I. (2017). Uso de las plataformas educativas y su impacto en la práctica pedagógica en instituciones de educación superior de San Luis Potosí. EDUTEC. Revista Electrónica de Tecnología Educativa, (60), 1-13. https:// doi.org/10.21556/edutec.2017.60

Rojas, N., Pérez, F., Torres, I., \& Peláez, E. (2014). Las aulas virtuales: una opción para el desarrollo de la educación médica. EDUMECENTRO, 6(2), 231-247.

Sierra, C. A. (2011). La educación virtual como favorecedora del aprendizaje autónomo. Panorama, 5(9), 75-87.

Sociedad Mexicana de Criminología Capítulo Nuevo León, A. C. (2020). Equipo de trabajo. https://www.somecrimnl.org/

Sociedad Mexicana de Criminología Capítulo Nuevo León, A. C. (2020). Revista. https://acspyc.es.tl/

Terrazas, R., \& Silva, R. (2013). La educación y la sociedad del conocimiento. Perspectivas, 16(32), 145-168.

Trinchet, C., \& Trinchet, R. M. (2007). La definición del problema: el paso primero y fundamental del proceso de investigación científica. Acimed, 16(2).

Vargas-Cubero, A., \& Villalobos-Torres, G. (2018). El uso de plataformas virtuales y su impacto en el proceso de aprendizaje en las asignaturas de las carreras de Criminología y Ciencias Policiales, de la Universidad Estatal a Distancia de Costa Rica. Revista Electrónica Educare, 22(1), 1-20. https://doi.org/10.15359/ree.22-1.2

Villagómez, J. A., Mora, Á. H., Barradas, D. S., \& Vázquez, E. (2014). El análisis FODA como herramienta para la definición de líneas de investigación. Revista Mexicana de Agronegocios, 35, 1121-1131. 